OPEN ACCESS

Edited by:

Dewei Chu,

University of New South

Wales, Australia

Reviewed by:

Zunfeng Liu,

Nankai University, China

Xuhui Sun,

Soochow University, China

*Correspondence:

Zhenzhong Hou

hzzhong1981@yeah.net

Laixia Yang

867368760@qq.com

Specialty section:

This article was submitted to

Energy Materials,

a section of the journal

Frontiers in Materials

Received: 29 December 2020

Accepted: 09 March 2021

Published: 01 April 2021

Citation:

Hou Z, Lu H, Li Y, Yang L and Gao Y

(2021) Direct Ink Writing of Materials

for Electronics-Related Applications: A

Mini Review. Front. Mater. 8:647229.

doi: 10.3389/fmats.2021.647229

\section{Direct Ink Writing of Materials for Electronics-Related Applications: A Mini Review}

\author{
Zhenzhong Hou ${ }^{1 *}$, Hai $\mathrm{Lu}^{1}$, Ying $\mathrm{Li}^{1}$, Laixia Yang ${ }^{2,3 *}$ and Yang Gao ${ }^{3}$ \\ ${ }^{1}$ College of Materials Science and Engineering, Xi'an University of Science and Technology, Xi'an, China, ${ }^{2}$ State Key \\ Laboratory for Manufacturing Systems Engineering, Xi'an Jiaotong University, Xi'an, China, ${ }^{3}$ College of Mechanical \\ Engineering, Xi'an University of Science and Technology, Xi'an, China
}

Recently, the fabrication of electronics-related components via direct ink writing (DIW) has attracted much attention. Compared to the conventionally fabricated electronic components, DIW-printed ones have more complicated structures, higher accuracy, improved efficiency, and even enhanced performances that arise from well-designed architectures. The DIW technology allows directly print materials on a variety of flat substrates, even a conformal one, well suiting them to applications such as wearable devices and on-chip integrations. Here, recent developments in DIW printing of emerging components for electronics-related applications are briefly reviewed, including electrodes, electronic circuits, and functional components. The printing techniques, processes, ink materials, advantages, and properties of DIW-printed architectures are discussed. Finally, the challenges and outlooks on the manufacture of 3D structured electronic devices by DIW are outlined, pointing out future designs and developments of DIW technology for electronics-related applications. The combination of DIW and electronic devices will help to improve the quality of human life and promote the development of science and society.

Keywords: electronics, ink material, electrode, flexible device, direct ink writing

\section{INTRODUCTION}

As the development of high technology, traditional electronic products with the disadvantages of bulky size, single function and high cost are gradually replaced by novel electronic ones that are portable and powerful. The important development trends of the next generation of electronics are light-weight, multi-functional and flexible. Among many material-processing technologies, 3D printing, also known as additive manufacturing, has driven great innovations in electronics due to its excellent machining accuracy and controllability (Kong et al., 2014; Muth et al., 2014). This technique creates complex objects and patterning layer by layer directly from a computer aided design, offering a flexible, efficient, low-cost and completely new bottom-up manufacturing strategy to fabricate electronic devices.

Among all of the 3D printing technologies, DIW has excellent ability to construct various 3D structures on the basis of direct extrusion and deposition of materials. More specifically, the DIW is a printing method to construct geometries layer-by-layer through the extrusion from the moving dispenser nozzle and the deposition on the substrate (Ambrosi and Pumera, 2016), and different driven forces liking pneumatic, piston, and screw can be applied to drive the ink dispensing 
(Malda et al., 2013). The related equipment and operation can refer to the review by Tian and coworkers (Tian et al., 2017). It is obviously that the direct writing parameters (pressure, speed, and nozzle size) and printing environments (temperature, direct writing medium) have a significantly influence on the DIW process. Only proper parameters and environments can make the printed ink to form a stable 3D structure. Due to the simple working principle, such a DIW equipment can be assembled easily by labs themselves, consisting of a dispenser, nozzle, three-axis platform, and computer (Guo et al., 2017). DIW is becoming the most versatile way to prepare $3 \mathrm{D}$ electrodes, $3 \mathrm{D}$ electronic components, and even integrated devices because of the facile printing strategy and fabrication process. Besides, a broad selection of raw materials covering metals, ceramics, polymers, and composites, is another feature considering the employment of DIW.

In fact, many review articles about DIW technology have been published. Some of them involve the application of DIW in electronics, but the corresponding discussion is relatively simple and not clear enough. Therefore, we tries to discuss recent advancements of DIW for electronics-related applications more clearly and comprehensively, including electrodes, electronic circuits, and functional components. Finally, we discuss a conclusion and outlook, expecting that this mini review could promote the further developments and innovations of DIW for electronics-related applications.

\section{MATERIAL CONSIDERATIONS}

Printable inks are the most important component for DIW, and directly affect the resolution, conductivity and mechanical properties of the electronics-related devices architectures. The material must be designed to the suitable ink which can flow smoothly through the dispenser nozzle without any clogging. Generally, two important criteria need to be mat for formulating the printable inks. One is high shear-thinning behavior and viscoelasticity (Fu et al., 2017; Pinargote et al., 2020). With this characteristic, the ink, as its reduced viscosity under driven force, is extruded easily from the dispenser in the form of a continuous rod-like filament, and then exhibits rapid "curing" to maintain a stable shape without collapse because of its sufficiently high yield stress and storage modulus. The other is a high solid content of the ink. This can avoid, as far as possible, the changes of volume and shape of the printed object during the following treatment (Lewis, 2006; Tian et al., 2017). The above two criteria require a proper control of ink formulations and rheological properties to form a stable dispersion that promotes the transition from fluid to gel, which ensures the fusion with previously deposited layers and retention of the printed shapes at the same time. The inks, mainly including sol-gels, polymer melts, wax-based materials and polyelectrolyte, usually have the composite modulus of $10^{3}-10^{5} \mathrm{~Pa}$, and the storage modulus $\left(\mathrm{G}^{\prime}\right)$ is higher than the loss modulus $\left(\mathrm{G}^{\prime \prime}\right)$. To optimize the rheology of inks as well as improving their electrochemical, conductive and mechanical properties, adding nano-materials as fillers has been proven to be an efficient way. For example, graphene oxide (GO) (Shen et al., 2018; Sun et al., 2020), silane-treated hexagonal boron nitride nanosheets (Cheng et al., 2020), $\mathrm{TiO}_{2}$ nanoparticles (Blake et al., 2017), $\mathrm{Al}_{2} \mathrm{O}_{3}$ nanoparticles (Cheng et al., 2018), and $\mathrm{Pt}$ nanoparticles (Nguyen et al., 2019) as fillers can not only tune the rheological properties of inks, but also significantly improve their electrical conductivity, thermal conductivity, ionic conductivity, and electro-activity, respectively.

Actually, the materials preparation and printing parameters (ink exiting speed, nozzle size, dispenser moving speed, ambient temperature, and direct writing medium) should be both considered at the beginning of DIW. Although these considerations have been widely investigated and addressed in some applications, the understanding in electronics-related applications is still in the early stage, which requires a systematical study to obtain desired electronic devices.

\section{DIW FOR ELECTRONICS-RELATED APPLICATIONS}

\section{Electrodes}

Electrodes are indispensable parts of some electronic devices. It is easy to prepare them with common structures but difficult to fabricate them with special structures by traditional methods. In order to meet the requirements of precision forming and high performance of electronic devices, the researchers use DIW to print electrodes with special structures.

Zhu et al. used the GO/silica powders suspension as the ink to print 3D periodic structures (Zhu et al., 2015). After supercritical drying and carbonization, this printed GO structure was converted to a $3 \mathrm{D}$ periodic graphene aerogel microlattice with high specific surface area and conductivity of $704 \mathrm{~m}^{2}$ $\mathrm{g}^{-1}$ and $278 \mathrm{~S} \mathrm{~m}^{-1}$, respectively. Lacey et al. added holey graphene oxide (hGO) to green solvents, such as water, to make additive-free inks with appropriate rheology, which was employed to print $\mathrm{Li}-\mathrm{O}_{2}$ cathodes with the similar structures to Zhu et al. (2015), as shown in Figures 1A,B (Lacey et al., 2018). As the hGO was inherently nanoporous with $4-25 \mathrm{~nm}$ through-holes, the printed $\mathrm{Li}_{-} \mathrm{O}_{2}$ cathodes had multi-sized pores from nanoscale (through-holes on hGO sheets) to microscale (lyophilization-induced micropores on the printed filaments) to macroscale (pores of mesh design) (Figure 1B). This hierarchical porosity was benefit for transfer of oxygen, electrolytes and ions. After the thermal reduction, the printed $3 \mathrm{D}$ electrode based on reduced hGO showed a much higher areal capacitance of $13.3 \mathrm{mAh} \mathrm{cm}^{-2}$ at $0.1 \mathrm{~mA} \mathrm{~cm}{ }^{-2}$ than the traditional $2 \mathrm{D}$ electrodes ( $\mathrm{Lu}$ et al., 2020; Yuan et al., 2020). Tang et al. printed graphene and its mixed-dimensional hybrid aerogels using graphene-based mixed-dimensional $(2 \mathrm{D}+\mathrm{nD}, n=0,1$, or 2) hybrid materials as inks, overcoming the limitations of multi-components inhomogeneity and harsh post-treatments for additives removal (Tang et al., 2018). The coin-type symmetric supercapacitor with the printed hybrid aerogels as electrodes exhibited the maximum areal capacitance of $639.56 \mathrm{mF} \mathrm{cm}^{-2}$ at $4 \mathrm{~mA} \mathrm{~cm}^{-2}$ and capacitance retention of $71.4 \%$ at $40 \mathrm{~mA} \mathrm{~cm}^{-2}$. The good capacitance and rate performance are attributed to the unique structure of 3D scaffolds of the printed electrodes, which 

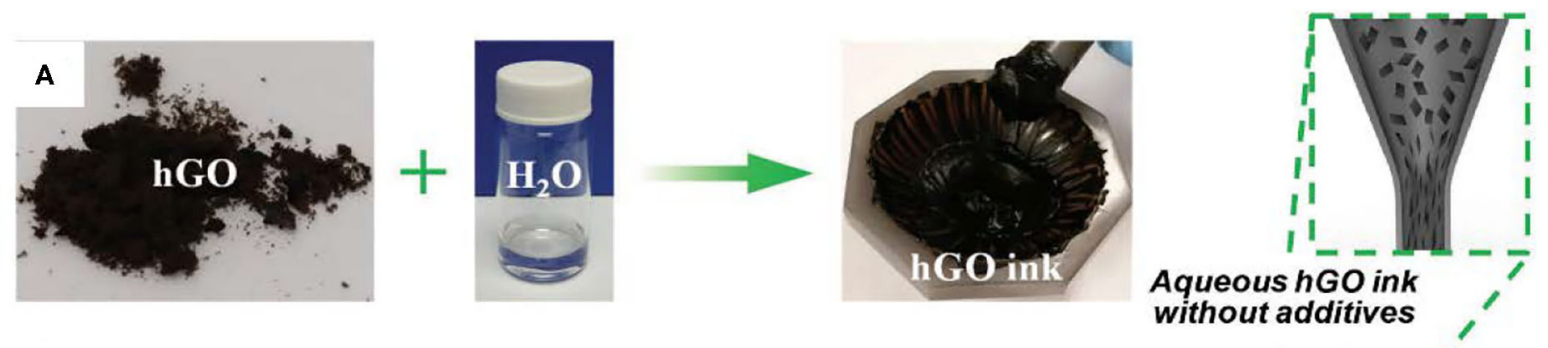

B

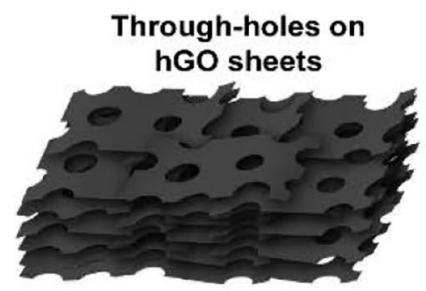

Nanoscale Porosity (Tens of nanometers)
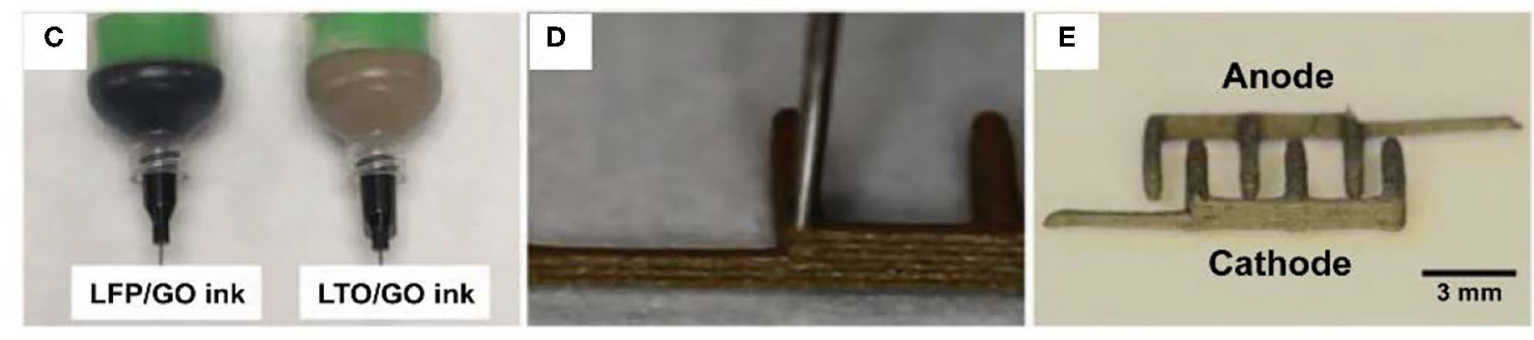

F

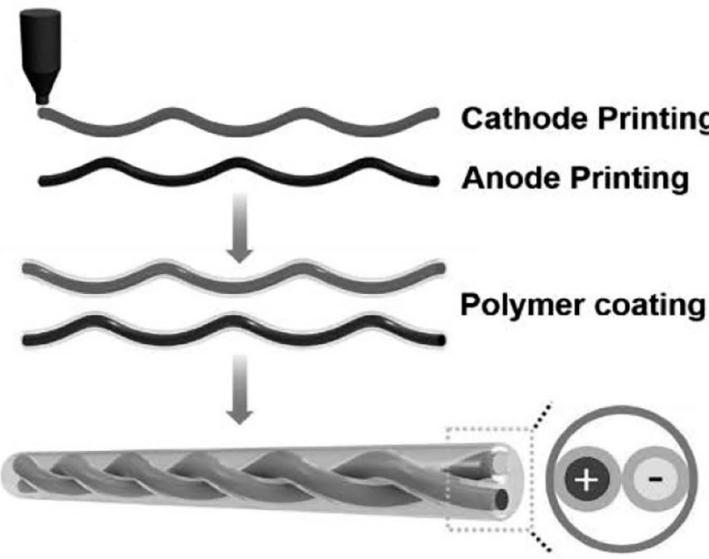

3D Printed All-Fiber LIBs

Lyophilization-induced micropores on the printed filaments

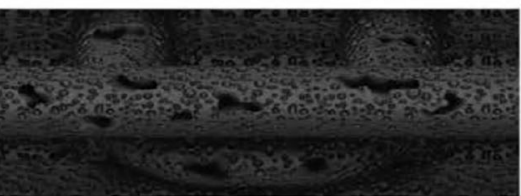

Microscale Porosity

(Tens of microns)
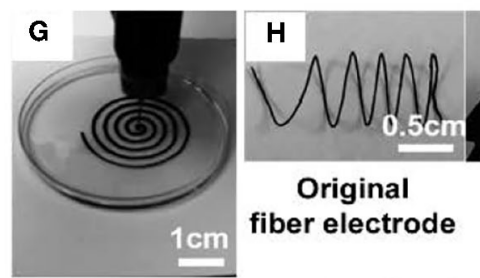

Original fiber electrode
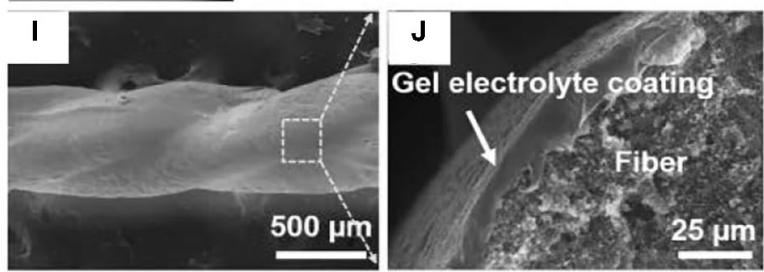

FIGURE 1 | (A) Schematics of making additive-free printable hGO ink. (B) Printing 3D scaffolds electrode with hierarchical porosity. Reproduced from Lacey et al. (2018) with permission from Wiley. (C) Digital images of the LFP/GO and LTO/GO inks. (D) Digital image of layer by layer printing process. (E) Digital image of printed interdigitated electrodes. Reproduced from Fu et al. (2016) with permission from Wiley. (F) Schematic of the design concept and fabrication process of all-fiber flexible Li-ion batteries. (G) Printing the fiber electrode process. (H) Digital images of the dry fiber electrodes. (I) SEM image of yarn fibers with polymer electrolyte coating. (J) Cross-sectional SEM image of the polymer electrolyte coating fibers. Reproduced from Wang et al. (2017) with permission from Wiley.

can provide sufficient ion- and electron-transport paths from the bottom to top surface.

The interdigitated electrodes, being suitable for microelectronic devices, also can be prepared by the DIW technology. Fu et al. developed LFP $\left(\mathrm{LiFePO}_{4}\right) / \mathrm{GO}$ and LTO
$\left(\mathrm{Li}_{4} \mathrm{Ti}_{5} \mathrm{O}_{12}\right) / \mathrm{GO}$ composite inks to printed the interdigitated cathode and anode electrodes for Li-ion microbattery, respectively (Figures 1C-E) (Fu et al., 2016). The LFP and LTO nanoparticles distributed homogeneously in the GO matrix, ensuring good contact, electrical conductivity and mechanical 
integrity of electrodes. After thermal treatment, the two printed electrodes delivered stable cycling performance with specific capacities of about $160 \mathrm{mAh} \mathrm{g}^{-1}$ for LFP cathode and $170 \mathrm{mAh}$ $\mathrm{g}^{-1}$ for LTO anode, respectively. Sun et al. reported patterning viscous GO-based ink as interdigitated microelectrodes via DIW on the PET (polyethylene terephthalate) film, and forming the symmetrical micro-supercapacitor (Sun et al., 2015). It should be noted that the formed supercapacitor on PET showed a capacitance of up to $19.8 \mathrm{mF} \mathrm{cm}^{-2}$ and excellent flexibility, mechanical robustness and cycling stability.

In order to further facilitate the portability of electronic devices, the development of light, thin, flexible and wearable devices has become a hot research topic. Therefore, it is very important to prepare fiber electrodes easily and efficiently. Wang et al. carried DIW to print the fiber cathode and anode with mushy inks composed of LFP or LTO particles, polyvinylidene fluoride (PVDF, as binder) and carbon nanotubes (CNTs, as conductor) (Wang et al., 2017). As shown in Figures 1F-J, the printed fibers were twisted together with gel polymer as the electrolyte to assemble an all-fiber quasi-solid-state Liion battery, which demonstrated high flexibility, strength and electrochemical stability. Zhao et al. assembled a fiber asymmetric supercapacitor by using the DIW-printed $\mathrm{CNTs} / \mathrm{V}_{2} \mathrm{O}_{5}$ fiber cathode and CNTs/VN (vanadium nitride) fiber anode (Zhao et al., 2018). Its highest areal energy density $\left(41.28 \mu \mathrm{Wh} \mathrm{cm}^{-2}\right)$ and power density $\left(480 \mu \mathrm{W} \mathrm{cm}^{-2}\right)$ obviously exceeded some fiber supercapacitors produced by other method (Kou et al., 2014; Wang et al., 2015; Xu et al., 2015). The above mentioned fiber electrodes can be potentially integrated into textile fabrics for future wearable electronic applications. In short, DIW provides a facile, fast, cost-effective and scalable strategy to prepare the electrodes which are difficultly fabricated by traditional methods, opening up new opportunities for smart, sophisticated, miniaturized, 3D integrated, and wearable electronic devices.

\section{Electronic Circuits}

As a promising technology, printed electronics can fabricate electronic devices used in various fields, such as energy conversion and storage, flexible electronics, sensors and so on, attracting wide attentions. DIW represents an advanced and interesting method to replace traditional lithographic circuits due to its simple process, low cost, and digital patterning ability.

Zhang et al. melt-blended reduced graphene oxide ( $\mathrm{rGO}$ ) and poly(lactic acid) (PLA) as printable inks for printing 3D circuits, shown in Figures 2A-D (Zhang et al., 2016). The rGO/PLA composite ink had much improved mechanical properties in favor to maintain the printed structure and increase resolution, which were proved by the printed circuits with clear boundaries and sharp corners (Figures 2B,C). The printed circuits displayed excellent flexibility, as well as adhesion to the substrate without peeling off even under the bending state, and could be used to light up LEDs. Similarly, Abas et al. carried polyvinyl alcohol to modify the commercial carbon paste ink to improve its mechanical strength and adhesiveness, achieving high-quality micro-printing (Abas et al., 2019). Unfortunately, addition of the functional polymer greatly decreases the overall conductivity of the printed circuits. Hu et al. developed a particle-free composite ink which mainly consists of soluble silver salt and adhesive rubber for DIW-preparing highly conductive stretchable circuits (Hu et al., 2016). The ink was transparent solution with good chemical stability, and could be directly handwrote on various substrates to form well adhesive written traces. After in situ reduction, the traces turned into highly conductive circuits because of the formation of silver nanoparticles from high-loading silver salt in the ink. A heart-like circuit and pressure-sensitive switch were fabricated to present their working capability. In order to evaluate the printing quality, Tricot and coworkers developed a DIW equipment with a screw dispenser and silver paste ink (Electrodag 418SS from Henkel) to print circuits on commercial substrates or 3D objects, and compared with the conventional screen printing process (Tricot et al., 2018). The results showed DIW permitted printing patterns with a similar quality to the screen printing process but with a better regularity of the edges of printing lines. Besides, DIW saved more inks because of some ink remaining on the screen meshes after the squeegee stroke during screen printing. In the near future, integrating complex and fine circuitry on any $3 \mathrm{D}$ object will be realized.

Since high conductivity and nontoxicity, room-temperature liquid metals namely gallium and its alloys are important raw materials in the printed electronics field. Because of the wettability limitation of common gallium-based alloys to paper, only the polymer can be used as the printed substrate in various printing technology (Zheng et al., 2013; Gozen et al., 2014; Parekh et al., 2016; Wang et al., 2016). However, common paper is a low-cost platform for manufacturing flexible, lightweight and biodegradable devices. So far, there are still few methods to print gallium-based alloys on common paper, creating complex pattern of liquid metal on paper reserves a challenging. If the liquid metal can be stably and evenly spread on the paper surface, it can be effectively used to print paper-supported circuits. Recently, researchers have exploited two directions to achieve direct writing of liquid metal on paper. One is tuning the properties of alloy inks by adding additives. Chang et al. mixed liquid Ga75.4 $\operatorname{In}_{24.5}$ alloy and nickel powder to form a sticky ink, which possessed excellent plasticity and stable adhesivity to printer paper (Chang et al., 2018). The oxide film wrapping the nickel powder formed a skeleton for additional oxidation products, resulting in superior adhesion to paper. The other is modifying paper surface so that the printed liquid alloy can be firmly attached on paper. Wang et al. wetted the paper surface by microdroplets to reduce the roughness of paper and enhance the interface adhesion between liquid alloy $\left(\mathrm{Ga}_{75.4} \mathrm{In}_{24.5}\right)$ and paper, ensuring a smooth direct writing process (Wang et al., 2019). The two works provide a simple avenue to design and manufacture paper-based electronics.

Besides, other types of conductive materials are also used to make inks. Chen et al. introduced polypyrrole into graphene/carbon black composite, using a mixture of alcohol, ethylene glycol, glycerol and deionized water as solvent, to obtain a conductive ink with excellent water and acid resistance (Chen Y. et al., 2019). Uniform and flexibility 3D circuits were prepared by directly writing this ink on high-gloss photographic paper. It has been confirmed that, through distributing on 

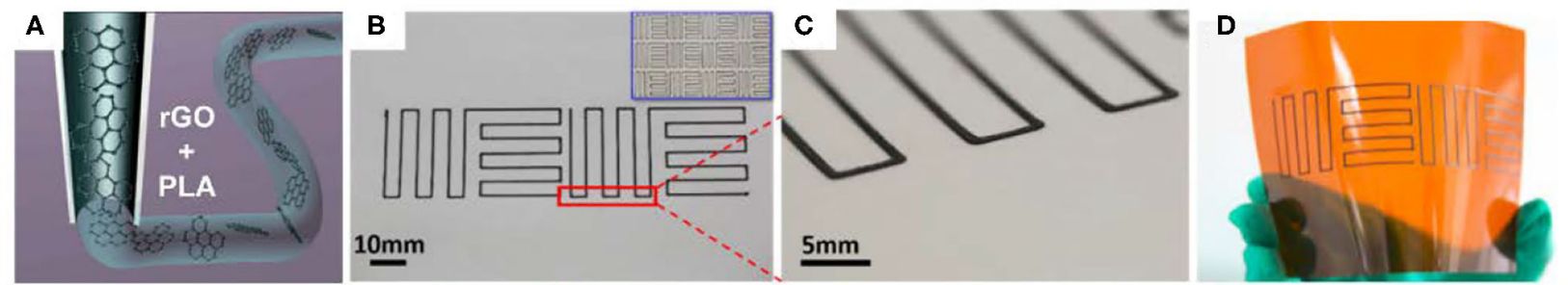

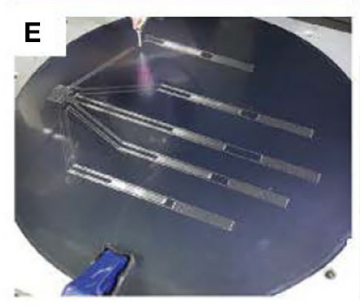

Printing

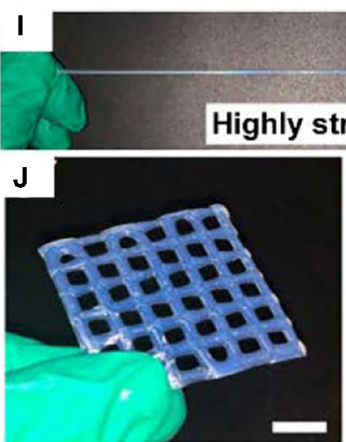

M

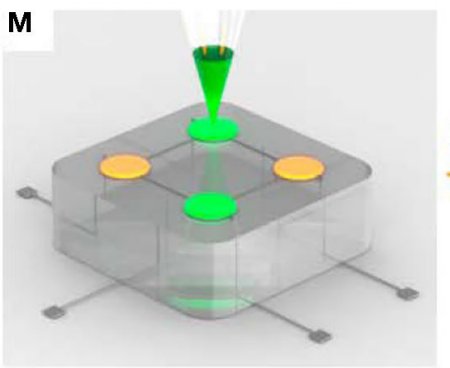

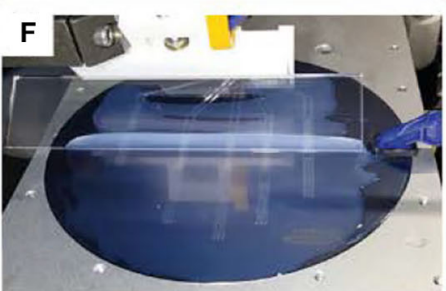

Encapsulating

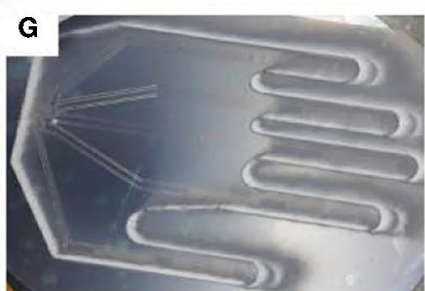

Laser cutting

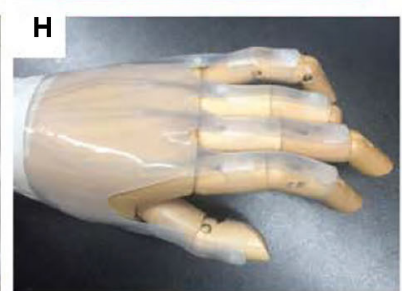

Attaching a glove sensor on a hand mock-up

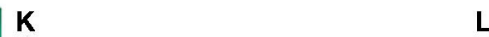

L
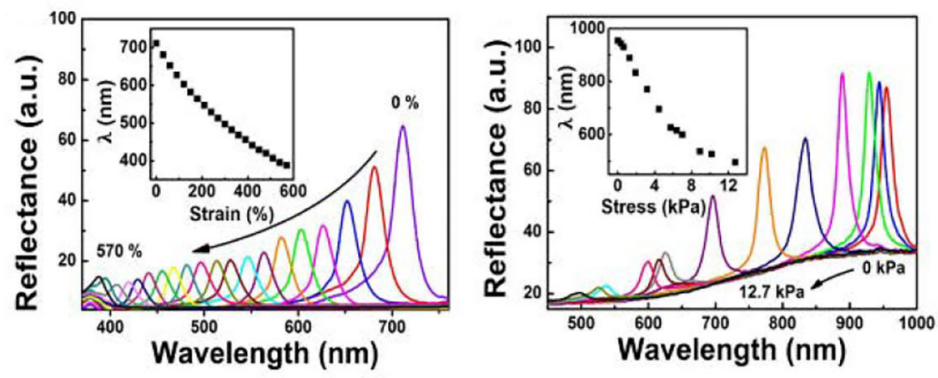

$\mathbf{N}$
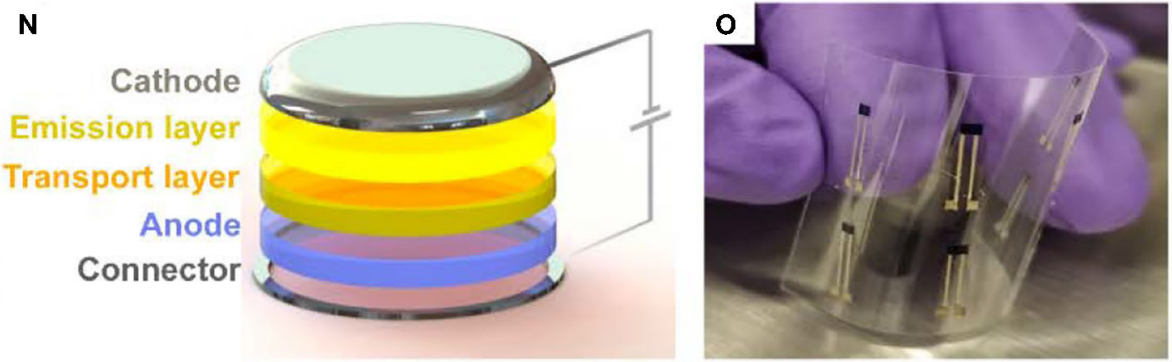

FIGURE 2 | (A) Schematic of printing circuits with the rGO/PLA composite ink. (B,C) Printed paper-based flexible circuits. (D) Printed polyimide-based flexible circuits. Reproduced from Zhang et al. (2016) with permission from Elsevier. (E-H) Fabrication process of a glove-type sensor. Reproduced from Kim et al. (2018) with permission from Mary Ann Liebert Inc. (I) Highly stretchable photonic crystal hydrogel. (J) Printed flexible photonic crystal hydrogel grid. (K,L) Normal incident reflectance spectra of photonic crystal hydrogel under different tensile strains (K) and compressive stresses (L). Insets: tensile strain (K) and compressive stress (L) dependence of maxima ( $\lambda$ ). Reproduced from Chen J. et al. (2019) with permission from American Chemical Society. (M) DIW-printing QDLEDs. (N) Layer-by-layer schematic of the QDLED components. Reproduced from Kong et al. (2014) with permission from American Chemical Society. (0) Printed PET-based flexible OECTs. Reproduced from Fan et al. (2019) with permission from Elsevier.

graphene surface, carbon black particles can effectively reduce the agglomeration of graphene sheets as well as bridge them to form conductive paths, further significantly improving the conductivity of the ink (Li et al., 2015; Shukla et al., 2015; Ji et al., 2018). Gogotsi $Y$ et al. developed additive-free $\mathrm{Ti}_{3} \mathrm{C}_{2}$ MXene-inwater inks which can be written directly on various substrates, including paper, textile, wood and plastics (Quain et al., 2019). The written circuits could be dried at room temperature in air and displayed relatively high conductivity between carbon-based and metal nanoparticle inks.

\section{Functional Components}

In addition to preparing electrodes and circuits mentioned above, DIW is often employed to prepare other electronic functional components such as sensors, transistors, light emitting diodes, antennas, and so on. Vatani and Abas et al. all adopted the method of direct writing first and then packaging to fabricate layered resistance sensors (Vatani et al., 2015; Abas and Rahman, 2016). They first printed the designed sensing arrays with corresponding CNTs and carbon paste inks, and then encapsulated them in photocurable resin and PET to assemble 
the sensors, respectively. Based on this technology, the geometry of the sensing arrays can be controlled accurately so as to realize stable sensing performances. As a result, high-quality soft sensors with consistent sensing arrays could be manufactured repeatedly with liquid metals as the printable inks (Parekh et al., 2016; Kim et al., 2018). Especially for Kim et al. (2018), they directly printed a one-piece glove-type sensor containing 10 strain gauges to measure flexion and extension of the five fingers (Figures 2E-H), presenting a compact sensor system and short production time (within $15 \mathrm{~min}$ ). Shi et al. prepared aqueous ink based on polydimethylsiloxane (PDMS) submicrobeads/GO nanocomposite, which enabled high-resolution 3D DIW of strain sensors (Shi et al., 2019). Through finely tuning rheology, the ink can be easily extruded from nozzles as small as $50 \mu \mathrm{m}$ allowing for printing sensing arrays with unrivaled resolution. The obtained micron-scale pressure sensors exhibited high structure resolution $(100 \mu \mathrm{m})$, excellent sensitivity $\left(0.3 \mathrm{kPa}^{-1}\right)$, and broad operating range $(0-500 \mathrm{kPa})$.

Recently, some other types of sensing components have been developed via DIW. Chen and colleagues created a precursor ink, containing acrylamide, an ultraviolet initiator, and the poly(butyl acrylate) spheres $(160 \mathrm{~nm})$ swollen by 2-ethylhexyl acrylate monomers, to construct a mechanochromic sensor by a direct-writing technique coupled with UV-curing (Chen J. et al., 2019). The precursor was cured under UV-irradiation to form the photonic crystal hydrogel, an interesting photonic soft material. It showed a high elasticity and excellent mechanochromic property of which structural color changed reversibly with different strains (Figures 2I-L). Therefore, this hydrogel can be ink-written into various shapes for wearable optical devices, colorimetric tactile sensors, and full-color displays. Siebert et al. firstly directly wrote mixed metal microparticles arrays of commercially available $\mathrm{Fe}$ and $\mathrm{Cu}$ particles onto the glass substrate for sensing acetone gas (Siebert et al., 2020). After thermal annealing, the mixed metal microparticles formed open, highly porous nanostructures based on bridging $\mathrm{CuO} / \mathrm{Cu}_{2} \mathrm{O} / \mathrm{Cu}$ $\mathrm{Fe}_{2} \mathrm{O}_{3} / \mathrm{Fe}$ heterojunction networks, achieving high selectivity and sensitivity to acetone vapor at an operating temperature of $300^{\circ} \mathrm{C}$ and operating power as low as $0.26 \mu \mathrm{W}$.

In addition, Kong et al. utilized DIW to printed quantum dot light emitting diodes (QDLEDs), in which every component, including cathode, anode, transport layer, and light-emitting layer, is 3D printed (Figures $\mathbf{2 M}, \mathbf{N}$ ), exhibiting pure and tunable color emission properties (Kong et al., 2014). The QDLEDs could be directly constructed on the substrates with various shapes by incorporating a 3D light-scanning technique. The combination of DIW and 3D scanning technology makes it easier to copy and improve the existed structure, which paves a new way for efficiently preparing complex 3D integrated electronic devices. Fan et al. reported fully $3 \mathrm{D}$ printed organic electrochemical transistors (OECTs) by DIW with the poly $(3,4$ ethylenedioxythiophene)/poly(styrenesulfonate) composite and silver paste as the channel material and source/drain material, respectively (Figure 2O) (Fan et al., 2019). These printed devices demonstrated high transconductance and current ON/OFF ratio, low operating voltage, as well as excellent stability and robustness after several measurement cycles, being applicable for affordable and customized biosensing and biowearables.
Through photomask-assisted DIW, Chen et al. designed a multilevel triboelectric nanogenerator as an energy harvester to enlighten LEDs under external force, for self-powered wearable devices (Chen K. J. et al., 2019). With the Ag paste or Ga/In alloy ink, a hemispherical antenna with tunable resonant frequency could be DIW-printed, showing potential for wireless sensing and dynamic communication (Adams et al., 2011; Boley et al., 2019).

Obviously, using DIW technology to print functional components is expected to realize preparation and assembly of various components in the electronic equipment, simultaneously. The DIW-assembled components have higher molding accuracy and better compatibility, which will help to promote the development of fully printed electronic devices.

\section{SUMMARY AND OUTLOOK}

DIW technology provides a new strategy for the preparation of electronic devices, which can print structures with specific functions more conveniently and quickly, offering more possibilities for human beings to play their subjective activity and realize the transformation from mind to reality. Here, we have briefly reviewed the recent developments of DIW for electronicrelated applications based on different materials. Compared with the traditional electronic manufacturing technology, DIW technology not only has a strong 3D structure designability, but also is more gentle and convenient because of no irritant chemicals and high-temperature processing being required. Besides, the DIW equipment is simple, inexpensive, easy to assemble and operate, and less space occupation, so it has broad commercial prospects. However, there are still several challenges needed to be addressed. Firstly, the resolution, accuracy and surface finish of DIW need to be improved. So far, it is rather difficult to print in submicron scale by DIW. Secondly, the materials suitable for DIW-preparing electronics are still quite limited compared to the large number of materials extensively used in conventional processes. Thirdly, DIWprinting integrated electronic devices are difficult because of different materials based-components requiring different inks. Fourthly, a high-cost post-processing is commonly needed in a printing process and the efficiency is low. Therefore, three aspects of research are needed to address the above challenges in the future work. The first is designing and developing more printable conducting inks with the desired rheology (non-Newtonian paste with shear-shinning behavior), green solvent, and long storing time, that is a prerequisite to achieve high-resolution printing and expand printable materials. The second is creating advanced DIW equipment with higher resolution and capability of multi-materials printing, which is a guarantee to print integrated and miniaturized devices. The last is improving printing process and developing integrated printing systems to achieve post-processing-free fabrication and high efficiency.

In the future, DIW technology will continue to move toward diversification of the materials, structures and methods. It would be quite promising to combine emerging DIW processes with conventional fabrication techniques. Overall, DIW is expected to be a disruptive innovation that changes 
the way we think about fabricating and integrating electronics. The transformation of conventional electronics fabrication toward DIW innovative technology will lead to the next generation of highly embedded electronics, flexible devices, and wearable systems.

\section{AUTHOR CONTRIBUTIONS}

$\mathrm{ZH}$ wrote and supervised the manuscript. HL analyzed the references. YL and YG edited the manuscript. LY performed

\section{REFERENCES}

Abas, M., and Rahman, K. (2016). Fabrication of flex sensors through direct ink write technique and its electrical characterization. Appl. Phys. A 122:972. doi: 10.1007/s00339-016-0507-8

Abas, M., Salman, Q., Khan, A. M., and Rahman, K. (2019). Direct ink writing of flexible electronic circuits and their characterization. J. Braz. Soc. Mech. Sci. Eng. 41:563. doi: 10.1007/s40430-019-2066-3

Adams, J. J., Duoss, E. B., Malkowski, T. F., Motala, M. J., Ahn, B. Y., Nuzzo, R. G., et al. (2011). Conformal printing of electrically small antennas on three-dimensional surfaces. Adv. Mater. 23, 1335-1340. doi: 10.1002/adma.201003734

Ambrosi, A., and Pumera, M. (2016). 3D-printing technologies for electrochemical applications. Chem. Soc. Rev. 45, 2740-2755. doi: 10.1039/C5CS00714C

Blake, A. J., Kohlmeyer, R. R., Hardin, J. O., Carmona, E. A., Maruyama, B., Berrigan, J. D., et al. (2017). 3D printable ceramic-polymer electrolytes for flexible high-performance li-ion batteries with enhanced thermal stability. $A d v$. Energy Mater. 7:1602920. doi: 10.1002/aenm.201602920

Boley, J. W., van Rees, W. M., Lissandrello, C., Horenstein, M. N., Truby, R. L., Kotikian, A., et al. (2019). Shape-shifting structured lattices via multimaterial 4D printing. Proc. Natl. Acad. Sci. U. S. A. 116, 20856-20862. doi: 10.1073/pnas.1908806116

Chang, H., Guo, R., Sun, Z., Wang, H., Hou, Y., Wang, Q., et al. (2018). Direct writing and repairable paper flexible electronics using nickel-liquid metal ink. Adv. Mater. Interfaces 5:1800571. doi: 10.1002/admi.201800571

Chen, J., Xu, L., Yang, M., Chen, X., Chen, X., and Hong, W. (2019). Highly stretchable photonic crystal hydrogels for a sensitive mechanochromic sensor and direct ink writing. Chem. Mater. 31, 8918-8926. doi: 10.1021/acs.chemmater.9b02961

Chen, K. J., Zhang, L., Kuang, X., Li, V., Lei, M., Kang, G. Z., et al. (2019). Dynamic photomask-assisted direct ink writing multimaterial for multilevel triboelectric nanogenerator. Adv. Funct. Mater. 29:1903568. doi: 10.1002/adfm.201903568

Chen, Y., Zhou, L., Wei, J., Mei, C., Jiang, S., Pan, M., et al. (2019). Direct ink writing of flexible electronics on paper substrate with graphene/polypyrrole/carbon black ink. J. Electron. Mater. 48, 3157-3168. doi: 10.1007/s11664-019-07085-x

Cheng, M., Jiang, Y., Yao, W., Yuan, Y., Deivanayagam, R., Foroozan, T., et al. (2018). Elevated-temperature 3D printing of hybrid solid-state electrolyte for li-ion batteries. Adv. Mater. 30:1800615. doi: 10.1002/adma.201800615

Cheng, M., Ramasubramanian, A., Rasul, M. G., Jiang, Y., Yuan, Y., and Foroozan, T. (2020). Direct ink writing of polymer composite electrolytes with enhanced thermal conductivities. Adv. Funct. Mater. 20:2006683. doi: $10.1002 /$ adfm. 202006683

Fan, J., Montemagno, C., and Gupta, M. (2019). 3D printed high transconductance organic electrochemical transistors on flexible substrates. Org. Electron. 73, 122-129. doi: 10.1016/j.orgel.2019.06.012

Fu, K., Wang, Y., Yan, C., Yao, Y., Chen, Y., Dai, J., et al. (2016). Graphene oxide-based electrode inks for 3D-printed lithium-ion batteries. Adv. Mater. 28, 2587-2594. doi: 10.1002/adma.201505391

Fu, K., Yao, Y., Dai, J., and Hu, L. (2017). Progress in 3D printing of carbon materials for energy-related applications. Adv. Mater. 29:1603486. doi: 10.1002/adma.201603486 conceptualization and funding acquisition. All authors contributed to the article and approved the submitted version.

\section{FUNDING}

This work was funded by the Key Research and Development Program of Shaanxi Province (2017GY-133), the National Natural Science Foundation of China (51605379), and the State Key Laboratory for Manufacturing Systems Engineering (Xi'an Jiaotong University).

Gozen, B. A., Tabatabai, A., Ozdoganlar, O. B., and Majidi, C. (2014). Highdensity soft-matter electronics with micron-scale line width. Adv. Mater. 26, 5211-5216. doi: 10.1002/adma.201400502

Guo, S. Z., Qiu, K. Y., Meng, F. B., Park, S. H., and McAlpine, M. C. (2017). 3D printed stretchable tactile sensors. Adv. Mater. 29:1701218. doi: 10.1002/adma.201701218

Hu, M., Cai, X., Guo, Q., Bian, B., Zhang, T., and Yang, J. (2016). Direct pen writing of adhesive particle-free ultrahigh silver salt-loaded composite ink for stretchable circuits. ACS Nano 10, 396-404. doi: 10.1021/acsnano.5b05082

Ji, A., Chen, Y., Wang, X., and Xu, C. (2018). Inkjet printed flexible electronics on paper substrate with reduced graphene oxide/carbon black ink. J. Mater. Sci. Mater. Electron. 29, 13032-13042. doi: 10.1007/s10854-018-9425-1

Kim, S., Oh, J., Jeong, D., Park, W., and Bae, J. (2018). Consistent and reproducible direct ink writing of eutectic gallium-indium for high-quality soft sensors. Soft Robot. 5, 601-612. doi: 10.1089/soro.2017.0103

Kong, Y. L., Tamargo, I. A., Kim, H., Johnson, B. N., Gupta, M. K., Koh, T. W., et al. (2014). 3D printed quantum dot light-emitting diodes. Nano Lett. 14, 7017-7023. doi: 10.1021/nl5033292

Kou, L., Huang, T., Zheng, B., Han, Y., Zhao, X., Gopalsamy, K., et al. (2014). Coaxial wet-spun yarn supercapacitors for high-energy density and safe wearable electronics. Nat. Commun. 5:3754. doi: 10.1038/ncomms4754

Lacey, S. D., Kirsch, D. J., Li, Y., Morgenstern, J. T., Zarket, B. C., Yao, Y., et al. (2018). Extrusion-based 3D printing of hierarchically porous advanced battery electrodes. Adv. Mater. 30:1705651. doi: 10.1002/adma.201705651

Lewis, J. A. (2006). Direct ink writing of 3D functional materials. Adv. Funct. Mater. 16, 2193-2204. doi: 10.1002/adfm.200600434

Li, Y., Lu, X., Su, F., He, Y., Li, B., Yang, Q., et al. (2015). A graphene/carbon black hybrid material: a novel binary conductive additive for lithium-ion batteries. New Carbon Mater. 30, 128-132. doi: 10.1016/j.carbon.2015.04.071

Lu, H., Zhu, Y., Zheng, B., Du, H., Zheng, X., Liu, C., et al. (2020). A hybrid ionic liquid-based electrolyte for high-performance lithium-sulfur batteries. New J. Chem. 44, 361-368. doi: 10.1039/C9NJ03790J

Malda, J., Visser, J., Melchels, F. P., Jüngst, T., Hennink, W. E., Dhert, W. J. A., et al. (2013). 25th anniversary article: engineering hydrogels for biofabrication. $A d v$. Mater. 25, 5011-5028. doi: 10.1002/adma.201302042

Muth, J. T., Vogt, D. M., Truby, R. L., Meng,üç, Y., Kolesky, D. B., Wood, R. J., et al. (2014). 3D printing: embedded 3D printing of strain sensors within highly stretchable elastomers. Adv. Mater. 26, 6307-6312. doi: 10.1002/adma.201400334

Nguyen, T. N. H., Nolan, J. K., Park, H., Lam, S., Fattah, M., Page, J. C., et al. (2019). Facile fabrication of flexible glutamate biosensor using direct writing of platinum nanoparticle-based nanocomposite ink. Biosens. Bioelectron. 131, 257-266. doi: 10.1016/j.bios.2019.01.051

Parekh, D. P., Ladd, C., Panich, L., Moussa, K., and Dickey, M. D. (2016). 3D printing of liquid metals as fugitive inks for fabrication of 3D microfluidic channels. Lab Chip 16, 1812-1820. doi: 10.1039/C6LC00198J

Pinargote, M. W. S., Smirnov, A., Peretyagin, N., Seleznev, A., and Peretyagin, P. (2020). Direct ink writing technology (3D printing) of graphene-based ceramic nanocomposites: a review. Nanomaterials 10:1300. doi: 10.3390/nano10071300

Quain, E., Mathis, T. S., Kurra, N., Maleski, K., Van Aken, K. L., Alhabeb, M., et al. (2019). Direct writing of additive-free MXene-in-water ink for electronics and energy storage. Adv. Mater. Technol. 4:1800256. doi: 10.1002/admt.201800256 
Shen, K., Ding, J., and Yang, S. (2018). 3D printing quasi-solid-state asymmetric micro-supercapacitors with ultrahigh areal energy density. Adv. Energy Mater. 8:1800408. doi: 10.1002/aenm.201800408

Shi, G., Lowe, S. E., Teo, A. J. T., Dinh, T. K., Tan, S. H., Qin, J., et al. (2019). A versatile PDMS submicrobead/graphene oxide nanocomposite ink for the direct ink writing of wearable micron-scale tactile sensors. Appl. Mater. Today 16, 482-492. doi: 10.1016/j.apmt.2019.06.016

Shukla, S., Domican, K., Karan, K., Bhattacharjee, S., and Secanell, M. (2015). Analysis of low platinum loading thin polymer electrolyte fuel cell electrodes prepared by inkjet printing. Electrochim. Acta 156, 289-300. doi: 10.1016/j.electacta.2015.01.028

Siebert, L., Wolff, N., Ababii, N., Terasa, M. I., Lupan, O., Vahl, A., et al. (2020). Facile fabrication of semiconducting oxide nanostructures by direct ink writing of readily available metal microparticles and their application as low power acetone gas sensors. Nano Energy 70:104420. doi: 10.1016/j.nanoen.2019.104420

Sun, C., Liu, S., Shi, X., Lai, C., Liang, J., and Chen, Y. (2020). 3D printing nanocomposite gel-based thick electrode enabling both high areal capacity and rate performance for lithium-ion battery. Chem. Eng. J. 381:122641. doi: 10.1016/j.cej.2019.122641

Sun, G., An, J., Chua, C. K., Pang, H., Zhang, J., and Chen, P. (2015). Layerby-layer printing of laminated graphene-based interdigitated microelectrodes for flexible planar micro-supercapacitors. Electrochem. Commun. 51, 33-36. doi: 10.1016/j.elecom.2014.11.023

Tang, X., Zhou, H., Cai, Z., Cheng, D., He, P., Xie, P., et al. (2018). Generalized 3D printing of graphene-based mixed-dimensional hybrid aerogels. ACS Nano 12, 3502-3511. doi: 10.1021/acsnano.8b00304

Tian, X., Jin, J., Yuan, S., Chua, C. K., Tor, S. B., and Zhou, K. (2017). Emerging 3Dprinted electrochemical energy storage devices: a critical review. Adv. Energy Mater. 7:1700127. doi: 10.1002/aenm.201700127

Tricot, F., Venet, C., Beneventi, D., Curtil, D., Chaussy, D., Vuong, T. P., et al. (2018). Fabrication of $3 \mathrm{D}$ conductive circuits: print quality evaluation of a direct ink writing process. RSC Adv. 8, 26036-26046. doi: 10.1039/C8RA03380C

Vatani, M., Engeberg, E. D., and Choi, J. W. (2015). Conformal directprint of piezoresistive polymer/nanocomposites for compliant multi-layer tactile sensors. Additive Manuf. 7, 73-82. doi: 10.1016/j.addma.2014. 12.009

Wang, L., Wang, M., Lu, J., Ardhi, R. E. A., Liu, J., Liu, G., et al. (2019). Enhanced adhesion between liquid metal ink and the wetted printer paper for direct writing electronic circuits. J. Taiwan Inst. Chem. Eng. 95, 202-207. doi: 10.1016/j.jtice.2018.07.003
Wang, Q., Yu, Y., Yang, J., and Liu, J. (2016). Fast fabrication of flexible functional circuits based on liquid metal dual-trans printing. Adv. Mater. 27, 7109-7116. doi: 10.1002/adma.201502200

Wang, R., Lang, J., Zhang, P., Lin, Z., and Yan, X. (2015). Fast and large lithium storage in $3 \mathrm{D}$ porous $\mathrm{VN}$ nanowires-graphene composite as a superior anode toward high-performance hybrid supercapacitors. Adv. Funct. Mater. 25, 2270-2278. doi: 10.1002/adfm.201404472

Wang, Y., Chen, C., Xie, H., Gao, T., Yao, Y., Pastel, G., et al. (2017). 3D-printed all-fiber Li-ion battery toward wearable energy storage. Adv. Funct. Mater. 27:1703140. doi: 10.1002/adfm.201703140

Xu, P., Wei, B., Cao, Z., Zheng, J., Gong, K., Li, F., et al. (2015). Stretchable wireshaped asymmetric supercapacitors based on pristine and $\mathrm{MnO}_{2}$ coated carbon nanotube fibers. ACS Nano 9, 6088-6096. doi: 10.1021/acsnano.5b01244

Yuan, Y., Zheng, D., Lu, H., Zhu, Y., Li, Z., Qin, F., et al. (2020). A multifunctional gel coating design for simultaneous interface amelioration, polysulfide adsorption and redox regulation in lithium-sulfur batteries. Appl. Surf. Sci. 533:147490. doi: 10.1016/j.apsusc.2020.147490

Zhang, D., Chi, B., Li, B., Gao, Z., Du, Y., Guo, J., et al. (2016). Fabrication of highly conductive graphene flexible circuits by 3D printing. Synth. Met. 217, 79-86. doi: 10.1016/j.synthmet.2016.03.014

Zhao, J., Zhang, Y., Huang, Y., Xie, J., Zhao, X., Li, C., et al. (2018). 3D printing fiber electrodes for an all-fiber integrated electronic device via hybridization of an asymmetric supercapacitor and a temperature sensor. Adv. Sci. 5:1801114. doi: $10.1002 /$ advs. 201801114

Zheng, Y., Zhang, Q., and Liu, J. (2013). Pervasive liquid metal based direct writing electronics with roller-ball pen. AIP Adv. 3:112117. doi: 10.1063/1.4832220

Zhu, C., Han, T. Y., Duoss, E. B., Golobic, A. M., Kuntz, J. D., Spadaccini, C. M., et al. (2015). Highly compressible 3D periodic graphene aerogel microlattice. Nat. Commun. 6:6962. doi: 10.1038/ncomms7962

Conflict of Interest: The authors declare that the research was conducted in the absence of any commercial or financial relationships that could be construed as a potential conflict of interest.

Copyright (c) $2021 \mathrm{Hou}, \mathrm{Lu}$, Li, Yang and Gao. This is an open-access article distributed under the terms of the Creative Commons Attribution License (CC BY). The use, distribution or reproduction in other forums is permitted, provided the original author(s) and the copyright owner(s) are credited and that the original publication in this journal is cited, in accordance with accepted academic practice. No use, distribution or reproduction is permitted which does not comply with these terms. 